1773. Abel. Exploring

https://doi.org/10.30953/tmt.v2.30

Page 1 of 7

\title{
MaineHealth Care At Home: Exploring Details of the MaineHealth Telemedicine Program
}

Robert Abel

Editor's note: On March $16^{\text {th }}$ and $17^{\text {th }}, 2017$, Telehealth and Medicine Today convened a national conference of opinion leaders to discuss and debate "Technologies and Tactics Transforming Long-term Care." Following that lecture, Robert Abel who is the Chief Nursing Officer and Director of Palliative Care for MaineHealth Care at Home, a member of the MaineHealth system, answer pointed questions about running a home telehealth program.

TMT: In the skilled nursing facility are you putting in the technology or just the protocols? [00:00]

Mr. Abel: Both. They're not using telehealth, specifically. But they're using a tablet on their own system. But when we know the patient is going to be discharged, will bring our technology in so the patient becomes familiar with that before they go home.

\section{TMT: Among all the parameters to monitor, which is most important for your telehealth program? [00:16]}

Mr. Abel: One of the things we've really learned is weight is probably the single most important indicator for us to pay attention to. So, we want the weight to be done in the facility on our equipment for a couple of days so we're sure we have the right number.

\section{TMT: You mentioned COPD during your lecture. Where do you stand on that} initiative? [00:35]

Mr. Abel: Right now we are working on COPD (chronic obstructive pulmonary disease). We're going to use a similar protocol. We have something called the COPD Rescue Kit-a similar protocol to this with steroids, antibiotics, inhaler. 
1773. Abel. Exploring

https://doi.org/10.30953/tmt.v2.30

Page 2 of 7

For those of you who know hospice or have worked with hospice, this is a similar sort of concept-that we can have orders ahead of time, have protocols available. It's a little bit more difficult to get medications, but if you put your mind to it you can do it

\section{TMT: How are you connecting palliative care with telehealth? [01:04]}

Mr. Abel: We are also working on a big project with palliative care. We are doing virtual visits with palliative care and with our heart failure patients. We are connecting providers by this same equipment with our palliative care patients. But now we're going to start connecting palliative care provider visits.

And we have a pediatric palliative care program. There's no pediatric hospice in Maine. So we've had a couple of young teenagers who have their own tablets and kind of do these their own thing and manage their own health care.

\section{TMT: Are the patients using these devices at home? [01:41]}

Mr. Abel: It's the certified home health agency. So, most of our patients have a payer, and are admitted for an episode. We have a technician that goes out and sets up the equipment. This is a nurse's aide who is trained to do this. And then a nurse goes in within 24 hours and does some of the teaching.

The other piece of that is that the equipment does some of the teaching, and it does the reminding. So, it tells you that it's time to take your blood pressure, and it will even show you how to put the blood pressure cuff on.

Everything is Bluetooth. Nothing is connected to wires or anything like that. So that's a real positive in terms of particularly our older patients.

We're able to, for example, put the scale in the bathroom or the bedroom, wherever they would normally use it. The older equipment was cabled to wherever the machine was. It's a lot safer, and it's sort of a more normal kind of way of doing it. 
1773. Abel. Exploring

https://doi.org/10.30953/tmt.v2.30

Page 3 of 7

TMT: How closely do you follow the physician treatment parameters and what triggers the protocol? [02:31]

Mr. Abel: We will not admit a patient unless we have physician ordered, provider ordered, parameters. Part of that is that we need to know what you want us to do if you're outside the parameters.

Typically, if you're over weight we activate the protocol. The best correlation is weight with heart failure; but because our patients have other co-morbidities it's not unusual for somebody not to gain weight and their pulse oximetry to change because they have COPD. It's all those things together. So we do a full set of vital signs, typically seven days a week; and we have a nurse at a station receiving that data, as well is it directly going over to the medical records system.

\section{TMT: How do the tablets connect the system? [03:16]}

Mr. Abel: The tablets are enabled for Verizon or ATT just because that's a Maine issue. I mean you could be in downtown Portland and not be able to get Verizon in this building, but you can get it in this one. So our vendor set it up for both. There's nobody that's not been able to transmit.

\section{TMT: How do the nurses stay connected with patients? [03:37]}

Mr. Abel: Our palliative care nurses have video on their cell phones. They use it a lot for social service consults. But we use it a lot more for families.

So again, because of who we are and where we are, our cellphones are set up so that we basically can communicate. And we have a huge service area. We have the whole of southern Maine. I'm not the IT (information technology) guy, but when they got the phones, they got phones that would work across our whole service area.

TMT: What are the backgrounds of the telehealth staff? [04:05] 
1773. Abel. Exploring

https://doi.org/10.30953/tmt.v2.30

Page 4 of 7

Mr. Abel: All our telehealth staff have cardiac backgrounds. Our supervisor and director of that area are certified and have extensive inpatient cardiac experience. Part of it was looking for the right people to do this.

\section{TMT: What is the reporting structure? [04:22]}

Mr. Abel: They are employees of the home health agency, but the medical director of the heart failure clinic kind of has a dotted line in the same way we do that with palliative care. For example, we have a palliative care clinic. The director of that clinic is kind of a consultant to the agency. So it's that's kind of the model that we use.

TMT: How do your nursing care protocols compare to the protocols in the clinic? [04:45]

Mr. Abel: Our nursing care protocols are the same protocols as if the nurse worked in the clinic.

TMT: Did you hire new staff? [04:55]

Mr. Abel: We use existing staff.

\section{TMT: Who makes the decision to place a patient in the system? [05:00]}

Mr. Abel: The referral provider is typically making the assessment that they think this patient's going to be able to manage this kind of program.

We have some exclusion criteria. Certain kinds of behavioral health and dementia, for example, would be an exclusion.

With some qualifications, if there was a family member there to help or something we would do that. But if the patient's going to be independent they've got to be able to stand on the scale. That's been a little bit of a struggle. And where weight is so important, we have bariatric scales, but you've got to be able to stand on them. So there's some limitations to what you can do at home. 
1773. Abel. Exploring

https://doi.org/10.30953/tmt.v2.30

TMT: How does MaineHealth view its role? [05:37]

Page 5 of 7

Mr. Abel: MaineHealth sees itself as many service lines, and instead of intersecting, I would say we grid our service lines.

There's much more integration. We continue to have a heart failure work group with members from across the system and from various areas. So for example, IT is part of that. And education is part of that. So there are these big steering groups that meet, typically quarterly. Even though we've got great success with this, that group continues to meet. And so that's some of how it kind of all fits together.

\section{TMT: What is the latest telehealth home health protocol you are working on?} [06:12]

Mr. Abel: Now we're starting to work on atrial fibrillation. Can we do something like this? And what would anticoagulation look like with a model like this.

To give you an idea of kind of how we're going to move this forward, the point of it is really to keep everybody talking to each other. I would say some of it is can you be really transparent with each other. If I have a problem with the clinic, or if my staff has a problem with the clinic, are they OK with calling the clinic and saying that. More importantly, is the medical director of the clinic OK with calling my staff and not going at them but having a conversation about what could work better? And that's relationships. That's what makes this work. And yes, people do that. It's not always pleasant, but they figure it out and they move on because they all want to do the right thing for the patient. So, it's both.

\section{TMT: Are these patients in primary care or part of your clinic? [07:04]}

Mr. Abel: It's both. And so I think that is the second piece to this. We use this protocol as sort of the skeleton, if you will, for the model. There's lots of cardiologists that want nothing to do with it as it's written. We say to them take the protocol, look at it, mark it up, write on it, tell us what you want to do, and we'll put that into the system. 
So there is a practice in Southern Maine that doesn't want to do this at all. They have in the system, and that's what we do so as long as it's evidence based.

This is also on the Internet, so if you Google MaineHealth diuretic protocol or MaineHealth, I think it actually is heart failure. There's several documents that you would have access to that are public domain.

All the physician practices related to this are on Epic in their offices. All of the MaineHealth hospitals are on Epic, and the agency is on Epic. Epic is our go-to platform, and interestingly enough somebody mentioned Mass General earlier there's even sort of interoperability among Epic.

\section{TMT: What is the most important piece for making home health a success?} [08:03]

Mr. Abel: We've got to have a single med database. That's my prediction for the future if we don't have that we're never going be successful.

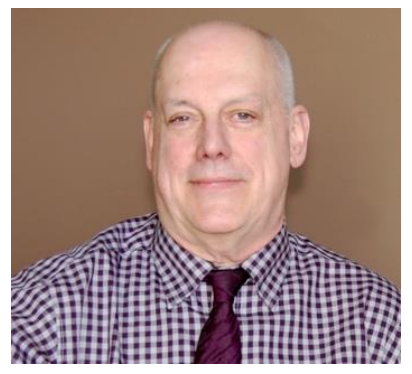

Robert Abel, MSN, RN, CHPN, CMC, CCM, is the Chief Nursing Officer for MaineHealth Care at Home. He has responsibility for the agency's telehealth program and involved in several system-wide initiatives to implement telehealth programs in areas such as heart failure, palliative care, and pediatrics. Mr. Abel previously worked with interdisciplinary clinical programs that used telehealth for decentralized, team-based, disease-driven care models. 
1773. Abel. Exploring

https://doi.org/10.30953/tmt.v2.30

Page 7 of 7

Tags: Robert Abel, COPD, atrial fibrillation, palliative care, communications, economics

of telehealth, governance, heart failure, home care, MaineHealth, Medicare, nursing care, sustainability, telehealth 